\title{
Pathohistological Diagnosis of Adrenal Tumors: Experience of a Single Center
}

Ivan Lekić ${ }^{1}$, Veronika Banović ${ }^{1}$, Ksenija Marjanović ${ }^{1,2}$, Borna Kovačić ${ }^{1,3}$, Ivan Feldi ${ }^{1,4}$, Tatjana Bačun ${ }^{* 1,5}$

1 Faculty of Medicine Osijek, Josip Juraj Strossmayer University Osijek, Croatia

2 Department of Pathology and Forensic Medicine, University Hospital Center Osijek, Croatia

3 Surgery Clinic, Department of Abdominal Surgery, University Hospital Center Osijek, Croatia

4 Department of Internal Medicine, Našice General Hospital, Našice, Croatia

5 Clinic for Internal Medicine, Department of Endocrinology, University Hospital Center Osijek, Croatia

*Corresponding author: Tatjana Bačun, tbacun@gmail.com

\begin{abstract}
Introduction: To investigate adrenal tumors of patients operated on at the University Hospital Center Osijek from 2016 to 2019 for the purpose of examining the location and histopathological findings of the tumors and determining whether there is a difference related to that in terms of the age and sex of the subjects.
\end{abstract}

Subjects and Methods: This was a cross-sectional study with historical data analysis. The subjects were patients of both sexes $(\mathrm{N}=23)$ diagnosed with adrenal tumors $(\mathrm{N}=23)$ who underwent surgery at the University Hospital Center Osijek in the period from 2016 to 2019. The archive was used in the University Hospital Center Osijek.

Results: Adrenal tumors were more often unilateral than bilateral. No differences were found in the localization of tumors of the right and left adrenal glands. Benign tumors were more common than malignant tumors and were the most common adenoma. Seventeen women underwent surgery and were 10 years older on average (61.6 years). There were no significant differences regarding tumor occurrence with respect to the patients' age. Benign tumors were more common in women, but the difference was not statistically significant. Regarding histopathological findings, women were more likely to have adenoma $(\mathrm{N}=10)$ and hyperplasia $(\mathrm{N}=4)$, while men had other benign tumors $(\mathrm{N}=3)$ and metastatic tumors $(\mathrm{N}=1)$. However, the difference was not statistically significant.

Conclusion: In patients who have undergone surgery for adrenal tumors, there were more unilateral tumors compared to bilateral ones, but there was no difference in tumor localization (left and right). Benign tumors were more common, the most common of which were adenomas. There were no significant differences with respect to age and sex.

(Lekić I, Banović V, Marjanović K, Kovačić B, Feldi I, Bačun T. Pathohistological Diagnosis of Adrenal Tumors: Experience of a Single Center. SEEMEDJ 2020; 4(2); 108-112)

Received: Apr 4, 2020; revised version accepted: Sep 28, 2020; published: Nov 12, 2020

KEYWORDS: histology, adrenal gland, adrenal tumor, adrenocortical adenoma, myelolipoma 


\section{Introduction}

The adrenal glands are bilateral glands located above each kidney, which typically weigh about 5 grams, but may be much larger in patients with chronic disease due to increased stimulation. The right adrenal gland is pyramid-shaped, while the left is crescent-shaped. The adrenal cortex in adults accounts for about $90 \%$ of the weight of the glands and there are three zones of the cortex: the glomerulosa zone, the fasciculata zone, and the reticularis zone. The neoplasms of the adrenal cortex can be benign (usually adenomas), malignant (cancers) and metastatic tumors. Furthermore, adrenal tumors can be hormone-active and inactive. Both functional and non-functional tumors occur more frequently in women than in men between the ages of 40 and 60 (1). Adenomas of the adrenal cortex are the most commonly benign lesions of the adrenal glands and the most common lesions in general (2). Adrenocortical cancers are extremely rare, with an incidence rate of one to two cases per million people per year, and as many as $15 \%$ of adrenal cancers are detected by chance. They have a bimodal distribution, that is, they appear in the first and fourth decade of life and are estimated to be functional in as many as $80 \%$ of cases, resulting in a characteristic clinical picture and in 55-60\% of cases involving the female sex (3). The adrenal tumors (adenomas and hyperplasia) are round, of well-circumscribed margins and a homogeneous structure. These tumors have visible separation from the surrounding tissues and do not branch into surrounding structures.

This study investigates the localization and pathohistological findings of adrenal neoplasms, as well as differences in terms of age and sex in subjects with adrenal tumors who underwent surgery at the University Hospital Center Osijek from 2016 to 2019.

\section{Patients and Methods}

The study involved an analysis of the medical records on patients diagnosed with adrenal tumors who underwent surgery at the
Department of Abdominal Surgery of the University Hospital Center Osijek from 2016 to 2019, which are kept by the Department of Abdominal Surgery of the University Hospital Center Osijek and the Clinical Institute of Pathology and Forensic Medicine. The data collected comprised: the patients' age and sex, diagnosis, tumor localization, pathohistological findings and the degree of pathohistological differentiation according to Weiss criteria. Adrenal tumor samples were analyzed morphologically using an Olympus ${ }^{\circledR} \quad \mathrm{CX}_{40}$ microscope on existing archival histological slides stained with the standard hematoxylin and eosin stain. The subjects were patients of both sexes diagnosed with adrenal tumors who underwent surgery at the University Hospital Center Osijek in the period from 2016 to 2019. The sample consisted of a total of 23 patients ( $N$ = 23). Samples of adrenal tumors were examined histologically at the Clinical Institute of Pathology and Forensic Medicine, University Hospital Center Osijek, which determined the degree of pathohistological differentiation according to Weiss criteria. The study used the medical records on patients diagnosed with adrenal tumors who were operated on at the Department of Abdominal Surgery of the University Hospital Center Osijek from 2016 to 2019, which are kept by the Department of Abdominal Surgery of the University Hospital Center Osijek and the Clinical Institute of Pathology and Forensic Medicine. The analysis was performed on existing archival histological slides using standard (hematoxylin and eosin) staining $(\mathrm{N}=23)$. Approval of the institutional ethical committee for this study was obtained from Faculty of Medicine Osijek.

\section{Statistical analysis}

In statistical analysis three tests were conducted. Fisher's Exact test, ANOVA test and Student's T-test. Fisher's Exact Test was used for statistical analysis of unilateral or bilateral adrenal neoplasms, malignancy between left or right neoplasms, pathological differences on adrenal neoplasms between sexes, localization of adrenal neoplasms between sexes. ANOVA 
test was used for statistical analysis for pathological diagnosis and age. Student's T-test was used for statistical analysis for age and sex and for age and size of adrenal neoplasms. $\mathrm{P}<0.05$ was set as a level of significance.

\section{Results}

Patients who have undergone surgery on for adrenal tumors at the University Hospital Center Osijek from 2016 to 2019 are shown. During this period, 23 patients were surgically treated. Tumors were more often unilateral $(\mathrm{N}=18)$ than bilateral $(N=5)$. No differences were found between tumor localization in the left $(\mathrm{N}=9)$ and right $(N=9)$ adrenal glands. In this study, it was observed that benign neoplasms had a higher incidence $(\mathrm{N}=22$ ) than malignant neoplasms ( $N$ = 1). Adrenal adenomas $(N=12)$ and hyperplasia $(\mathrm{N}=4)$ have the highest incidence of benign neoplasms, while only metastatic renal cancer was observed in malignant neoplasms $(N=1)$. The average age of the subjects was 58.9 (SD 11.6). The average age of men was 51.2 (SD 16.6). The average age of women was 61.6 (SD 8.3). It was observed that tumors occur in women at a later age, but the difference was not statistically significant (Student's T-test, $p=0.189$ ). The mean age of patients with unilateral tumors was 59.1 (SD 12.7) and with bilateral tumors 58.4 (SD 7.16). There was no statistically significant difference in adrenal tumor distribution in respect to age (data not shown) (Student's T-test, $p=0.914$ ). The average age of patients with tumors of the left adrenal gland was 63.6 (SD 9.96) and of the right adrenal gland 54.6 (SD 14.13), which was similar (ANOVA, $P=0.267$ ). The average age of individuals with adenoma was 60.3 (SD 9.17), with hyperplasia 61.5 (SD 6.14) and with other benign tumors 54.7 (SD 18.77). There was no statistically significant difference in respect to age and $\mathrm{PHD}$ of the tumor (ANOVA, $p=0.777$ ). Benign tumors were more common in women, but the difference was not statistically significant (Fisher's Exact Test, $p=0.261$ ). According to the histopathological findings of adrenal tumors, women had more frequent adenomas and hyperplasia, while benign tumors and metastases were more frequent in men, but the difference was not statistically significant (Fisher's Exact Test, $\mathrm{p}=0.110$ ).

The average tumor size was $4.87 \mathrm{~cm}$ (SD $1.95 \mathrm{~cm}$ ). The average tumor size in men was 5.77 $\mathrm{cm}$ (SD 2.42). The average tumor size in women was $4.55 \mathrm{~cm}$ (SD 1.73), which was similar between men and women. The average adenoma size was $4.098 \mathrm{~cm}$ (SD 1.22), hyperplasia 4.18 (SD 0.96), other benign tumors 7.22 (SD 1.91).

Patients with unilateral tumors were slightly older than patients with bilateral tumors. The patients with tumors of the left adrenal gland were slightly older than the patients with tumors of the right adrenal gland. The patients with adenoma and adrenal hyperplasia were older than patients with other benign adrenal changes. There was no correlation between age and histopathological diagnosis of the tumor (data not shown).

Women with adrenal tumors who were surgically treated were older than men. Men averaged 51.2 years of age (SD 16.6), while women averaged 61.6 years of age (SD 8.3), but the difference in age was not statistically significant.

\section{Discussion}

The study is a review of archival medical records kept by the Department of Abdominal Surgery of the University Hospital Center Osijek and the Clinical Institute of Pathology and Forensic Medicine from 2016 to 2019. The data were collected for 23 patients diagnosed with adrenal tumors who were operated on at the Department of Abdominal Surgery of the University Hospital Center Osijek. The data collected were analyzed to examine the existence of differences in localization (singlesided localization, left-right localization) and histopathological findings of tumors between subjects of both sexes. In our study, it was observed that unilateral neoplasms are more common than bilateral ones, which is consistent with the results of other studies $(1,4,10,11)$.

Data on tumor localization vary considerably in different studies and their samples. Mantero et al. and Kasperlik-Zaluska et

Southeastern European Medical Journal, 2020; 4(2) 
al. showed in their studies that neoplasms in the right adrenal gland were more common, unlike Bovio et al., who observed that tumors were more common in the left adrenal gland $(3,5,6)$. In our study, neoplasms of the right and left adrenal glands were equally common, which is in agreement with a similar study by Casola et al. (7).

In our study, obtained data suggest that benign adrenal neoplasms are the most common neoplasms (Figure 3), which is in agreement with a study by Szolar et al. and Cawood et al. (8.9). More than half of pathohistological diagnoses were adenomas, which is in line with other studies that cite adenomas as the most common pathohistological diagnosis $(8,9)$. Metastases are the most common malignant adrenal lesions and the second most common adrenal lesions in general (after adenoma) (2). The most common cancers that metastasize to the adrenal gland are cancers of the breast, lung, stomach, kidney and melanoma (10). In the case of multiple metastases, treatment is performed by methods other than solitary metastases only in the adrenal glands that are treated surgically (5). According to Angeli $A$ et al., women are more commonly affected by adrenal tumors $(1,2)$, which, in addition to the statistical significance, is shown by the results of this study based on the data from the University Hospital Center Osijek.

\section{References}

1. Angeli A, Osella G, Ali A, Terzolo $M$. Adrenal incidentaloma: an overview of clinical and epidemiological data from the National Italian Study Group. Horm Res 1997; 47:279.

2. Iñiguez-Ariza NM, Kohlenberg JD, Delivanis DA, Hartman RP, Dean DS, Thomas MA, Shah MZ, Herndon J, McKenzie TJ, Arlt W, Young Jr WF, Bancos I. Clinical, Biochemical, and Radiological Characteristics of a Single-Center Retrospective Cohort of 705 Large Adrenal Tumors. Mayo Clin Proc Innov Qual Outcomes 2018; 2:30.

3. Bovio S, Cataldi A, Reimondo G, Sperone P. Novello S, Berruti A, Borasio P, Fava C,
The risk of malignancy increases in proportion to the size of the tumor $(12,13)$. The best ratio of sensitivity to specificity is the tumor diameter of $4 \mathrm{~cm}$ (5).

Considering the limitations due to a small sample size used in our study, the value of this research is that it demonstrates our center's experience with histopathological findings of adrenal tumors. Monitoring needs to be continued in order to obtain data over a longer period.

Adrenal tumors were more often unilateral than bilateral, with equal incidence of the tumors of the right and left side, more often benign, most often adenomas. There was no significant difference with respect to age. Women had adenomas and hyperplasia more frequently, while other benign tumors and metastatic tumors were more frequent in men, but the difference was not statistically significant.

Acknowledgement. None.

\section{Disclosure}

Funding. No specific funding was received for this study.

Competing interests. None to declare.

Dogliotti L, Scagliotti GV, Angeli A, Terzolo M. Prevalence of adrenal incidentaloma in a contemporary computerized tomography series. J Endocrinol Invest 2006; 29:298.

4. Barzon L, Scaroni C, Sonino N, Fallo F, Gregianin M, Macri C, Boscaro M. Incidentally discovered adrenal tumors: endocrine and scintigraphic correlates. J Clin Endocrinol Metab 1998; 83:55.

5. Mantero F, Terzolo M, Arnaldi G, Osella G, Masini AM, Ali A, Giovagnetti M, Opocher G, Angeli A. A survey on adrenal incidentaloma in Italy. Study Group on Adrenal Tumors of the Italian Society of Endocrinology. J Clin Endocrinol Metab 2000; 85:637. 
6. Kasperlik-Zeluska AA, Rosłonowska E, Stowinska-Srzednicka J, Migdalska B, Jeske W, Makowska A, Snochowska H. Incidentally discovered adrenal mass (incidentaloma): investigation and management of 208 patients. Clin Endocrinol (Oxf) 1997; 46:29.

7. Casola G, Nicolet V, vanSonnenberg E, et al. Unsuspected pheochromocytoma: Risk of blood-pressure alterations during percutaneous adrenal biopsy. Radiology 1986; 159:733-735

8. Szolar $\mathrm{DH}$, Korobkin $\mathrm{M}$, Reittner $\mathrm{P}$, Berghold A, Bauernhofer T, Trummer $H$, Schoellnast $H$, Preidler KW, Samonigg $H$. Adrenocortical carcinomas and adrenal pheochromocytomas: mass and enhancement loss evaluation at delayed contrast-enhanced CT. Radiology 2005; 234:479.

9. Cawood TJ, Hunt PJ, O'Shea D, Cole D, Soule S. Recommended evaluation of adrenal incidentalomas is costly, has high false-positive rates and confers a risk of fatal cancer that is similar to the risk of the adrenal lesion becoming

\footnotetext{
' Author contribution. Acquisition of data: Lekić I, Banović V, Marjanović K, Kovačić B, Feldi I, Bačun T Administrative, technical or logistic support: Lekić I, Banović V, Marjanović K, Kovačić B, Feldi I, Bačun T Analysis and interpretation of data: Lekić I, Banović V, Marjanović K, Kovačić B, Feldi I, Bačun T

Conception and design: Lekić I, Banović V, Marjanović K, Kovačić B, Feldi I, Bačun T

Critical revision of the article for important intellectual content: Lekić I, Banović V, Marjanović K, Kovačić B, Feldi I, Bačun T

Drafting of the article: Lekić I, Banović V, Marjanović K, Kovačić B, Feldi I, Bačun T
}

malignant; time for a rethink? Eur J Endocrinol 2009; 161:513.

10. Young WF Jr. Clinical practice. The incidentally discovered adrenal mass. $\mathrm{N}$ Engl J Med 2007; 356:601.

11. Young WF Jr. Management approaches to adrenal incidentalomas. A view from Rochester, Minnesota. Endocrinol Metab Clin North Am 2000; 29:159.

12. Hamrahian $A H$, loachimescu AG, Remer EM, Motta-Ramirez G, Bogabathina H, Levin HS, Reddy S, Gill IS, Siperstein A, Bravo EL. Clinical utility of noncontrast computed tomography attenuation value (hounsfield units) to differentiate adrenal adenomas/hyperplasias from nonadenomas: Cleveland Clinic experience. J Clin Endocrinol Metab 2005: 90:871.

Grumbach MM, Biller BM, Braunstein GD, et al. Management of the clinically inapparent adrenal mass ("incidentaloma"). Ann Intern Med 2003; 138:424.

Final approval of the article: Lekić I, Banović V, Marjanović K, Kovačić B, Feldi I, Bačun T

Guarantor of the study: Lekić I, Banović V, Marjanović K, Kovačić B, Feldi I, Bačun T

Obtaining funding: Lekić I, Banović V, Marjanović K, Kovačić B, Feldi I, Bačun T

Provision of study materials or patients: Lekić I, Banović V, Marjanović K, Kovačić B, Feldi I, Bačun T Statistical expertise: Lekić I, Banović V, Marjanović K, Kovačić B, Feldi I, Bačun T

Other: Lekić I, Banović V, Marjanović K, Kovačić B, Feldi I, Bačun T 